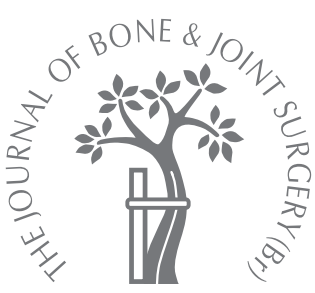

H. M. Betts, R. Abu-Rajab, T. Nunn, A. J. Brooksbank

From the Western Infirmary, Glasgow, Scotland

\title{
Total shoulder replacement in rheumatoid disease
}

\author{
A 16- TO 23-YEAR FOLLOW-UP
}

We describe the longer term clinical and radiological findings in a prospectively followed series of 49 rheumatoid patients (58 shoulders) who had undergone Neer II total shoulder replacement. The early and intermediate results have been published previously.

At a mean follow-up of 19.8 years (16.5 to 23.8 ) 14 shoulders survived. Proximal migration of the humeral component was associated with progressive loosening of the glenoid and humeral components, but was independent of the state of the rotator cuff at the time of operation. Despite these changes the range of movement was preserved. Most patients had little or no pain in the shoulder, could sleep undisturbed and could attend to personal hygiene and grooming.

Glenohumeral arthroplasty is widely accepted as a treatment of severe rheumatoid disease of the shoulder. Good short-term results have been achieved with the Neer II (3M, St. Paul Minnesota) total shoulder replacement (TSR), ${ }^{1-5}$ but there is ongoing concern about radiolucencies at the cement-bone interface of the glenoid component and their effect on pain and function in the longer term. ${ }^{6-8}$

There have been several studies on the longterm follow-up of patients with TSR, ${ }^{3,9,10}$ but the longest mean follow-up has been for 12.3 years. ${ }^{9}$ We now describe the longer term clinical and radiological findings in a prospectively followed group of rheumatoid patients with Neer II TSR. The early results in $42^{1}$ and the intermediate results in 32 of these patients ${ }^{2}$ have already been reported.

\section{Patients and Methods}

Between 1979 and 1985, 58 consecutive Neer II TSRs were carried out on 49 patients with seropositive rheumatoid arthritis. All the preoperative radiographs showed grade-IV or grade- $\mathrm{V}$ changes according to Larsen, Dale and Eek $^{11}$ at the glenohumeral joint. The indication for surgery was severe pain and marked limitation of function.

The operative technique and post-operative management have been described previously ${ }^{1}$ At operation, the state of the glenoid, humerus, deltoid and rotator-cuff tendons was recorded. All the glenoid components were cemented and all the humeral components were inserted without cement.
Of the original series, 29 patients (36 shoulders) had died, including one whose shoulder had been revised to an arthrodesis after a post-operative tear of the rotator cuff and one whose replacement was removed following infection. Three further survivors had undergone revision surgery. One implant had been removed for severe pain associated with loosening of the glenoid and humeral components and two had been revised for unknown reasons. One patient (one shoulder) was unable to attend for follow-up but provided clinical information. Four patients (four shoulders) were untraceable.

After a mean follow-up of 19.8 years $(16.5$ to 23.8 ), the remaining 12 patients (14 shoulders) were available for clinical and radiological review. There were 11 women (13 shoulders) and one man with a mean age at operation of 47.7 years (21.0 to 67.0). They scored their ability to carry out five activities of daily living and sleep on the replaced shoulder $^{1,2}$ and completed a visual analogue score (VAS) for pain.

Radiological evaluation. Anteroposterior and axillary radiographs of each shoulder were taken post-operatively, at six months, at one, two, and ten years and at the final review. Joint subluxation was assessed by the relationship of the junction of the middle and lower thirds of the prosthetic head to the centre of the wire marker on the glenoid component, with the arm at the side in neutral rotation. Subluxation was graded as none, mild $1<25 \%$ of the humeral head diameter), moderate $(25 \%$ to 
Table I. Radiological criteria for loosening of the glenoid component after total shoulder replacement

\begin{tabular}{ll}
\hline Grade & Criteria \\
\hline 0 & No loosening \\
1 & Radiolucency at superior and/or inferior flange \\
2 & Incomplete radiolucency at keel \\
3 & Complete radiolucency $(\leq 2 \mathrm{~mm}$ wide) around keel \\
4 & Complete radiolucency ( $>2 \mathrm{~mm}$ wide) around keel \\
5 & Gross loosening \\
\hline
\end{tabular}

Table II. Functional ability of the shoulder (including patient unable to attend for clinical review)

\begin{tabular}{lllc}
\hline & Pre-operative $(\mathbf{n}=\mathbf{1 5})$ & 2 years post-operative $(\mathbf{n}=\mathbf{1 5})$ & At latest follow-up (n = 15) \\
\hline Sleep on shoulder & 1 & 11 & 11 \\
Brush hair & 1 & 12 & 6 \\
Wash both axillae & 7 & 14 & 12 \\
Eat with utensils & 9 & 15 & 13 \\
Use hand at shoulder level & 0 & 12 & 7 \\
Perineal care & 3 & 13 & 6 \\
\hline
\end{tabular}

Table III. Mean $\left({ }^{\circ}\right.$, range) range of movement pre-operatively at two and 9.5 years post-operatively and at the latest follow-up (to nearest $5^{\circ}$ )

\begin{tabular}{|c|c|c|c|c|}
\hline \multirow[b]{2}{*}{ Range of movement ${ }^{*}$} & \multirow[b]{2}{*}{ Pre-operative } & \multicolumn{2}{|l|}{ Post-operative } & \multirow[b]{2}{*}{ Latest follow-up } \\
\hline & & 2 years & 9.5 years & \\
\hline LE & 45 (20 to 100$)$ & 75 (20 to 110$)$ & 75 (10 to 100$)$ & $60(0$ to 115$)$ \\
\hline ER & $5(-30$ to 30$)$ & $40(20$ to 70$)$ & $40(20$ to 50$)$ & $25(0$ to 50$)$ \\
\hline IR & Buttock (none - L5-S1) & L4 (trochanter - T12) & (Buttock - T7) & Buttock (none - L5-S1) \\
\hline
\end{tabular}

$50 \%$ of the humeral head diameter) or severe $(>50 \%) \cdot{ }^{1-4,12}$ As in the earlier reports we chose this method in preference to measurement of the acromiohumeral distance, which is very sensitive to radiological projection. ${ }^{1,2}$ Humeral loosening was defined as definite if there was subsidence, tilt or other change in position, or probable, if there were radiolucencies of more than $2 \mathrm{~mm}$ involving all or part of the stem or localised endosteal reaction.

The method of Franklin ${ }^{3,13}$ (Table I) was used to grade the degree of radiolucency around the keeled components and the overall seating of the glenoid component. According to this system, grade 3 would correspond to probable loosening in the previous reports of this group of patients. ${ }^{1,2}$ Statistical analysis was performed using Analyse-It for Excel (Microsoft, Redmond, Washington) The f-test was used to compare nominal data.

\section{Results}

Functional improvement showed some deterioration from that observed at the review at 9.5 years, although all the patients had better function than before operation. For 11 shoulders the patients were able to sleep on the affected shoulder compared with one pre-operatively and for 13 shoulders the patients continued to be able to eat with utensils using the affected limb compared with nine before operation (Table II).

The overall active range of movement had improved from that seen pre-operatively (Table III) but gradually decreased throughout the period of follow-up.

Radiological findings. The radiological results are shown in Table IV. There was a continuous radiolucent line around 12 glenoids (five grade 3 , four grade 4 and three grade 5 ). Two with complete lucent lines were noted to be loose at 9.5 years $^{2}$ and one of these had progressed to grade 5 . There was no significant relationship between the degree of glenoid loosening and the visual analogue scale (VAS) pain score $(p=0.63)$.

Upward migration of the humeral head occurred in all patients (two mild, six moderate and six severe). Loosening of the glenoid component was associated with proximal migration of the humeral component, with tip reaction and subsidence of the stem. A total of 11 humeral components showed signs of loosening, with radiolucencies around the stem or periosteal reaction at the top of the prosthesis (Fig. 1). Only one component had subsided and this had been noted previously at the follow-up at 9.5 years.

Most patients had little or no pain in the shoulder (Table IV), with a median VAS of 0.45 . Two reported severe pain, 
Table IV. Intra-operative findings, late radiological findings and visual analogue scale (VAS) scores for all shoulders

\begin{tabular}{clllllll}
\hline Case & $\begin{array}{l}\text { Glenoid at } \\
\text { operation }\end{array}$ & $\begin{array}{l}\text { Glenoid } \\
\text { lucency }\end{array}$ & Rotator-cuff & $\begin{array}{l}\text { Humeral } \\
\text { lucency }\end{array}$ & $\begin{array}{l}\text { Humeral } \\
\text { subluxation }\end{array}$ & $\begin{array}{l}\text { Proximal humeral } \\
\text { migration }\end{array}$ \\
\hline $1^{*}$ & Deficient & 3 & Thin & Tip & No & Mild & Moderate \\
$2^{*}$ & Normal & 3 & Normal & Tip & No & Moderate & 0.3 \\
3 & Deficient & 4 & Thin & Complete & No & Severe & 1.3 \\
4 & & 5 & & Neck/lateral & No & Severe & 0.0 \\
5 & Deficient & 2 & Minor tear & Tip & No & Severe \\
6 & Normal & 4 & Normal & Neck/tip & No & Moderate \\
7 & Deficient & 3 & Thin & Neck/tip & No & Moderate & 0.3 \\
8 & Deficient & 5 & Thin & Tip & No & Moderate \\
9 & Deficient & 3 & Normal & Tip & No & Mild \\
$10^{*}$ & Normal & 3 & Thin & Tip & No & Moderate \\
$11^{*}$ & Porotic & 2 & Minor tear & No & No & Severe \\
12 & Normal & 4 & Thin & Tip & No & Severe \\
13 & Deficient & 4 & Thin & Neck/tip & Yes & Severe \\
14 & Deficient & 5 & Thin & Neck/tip & 0.0 \\
\hline
\end{tabular}

* Bilateral total shoulder replacement

† Patient with intraoperative fracture at tip of prosthesis

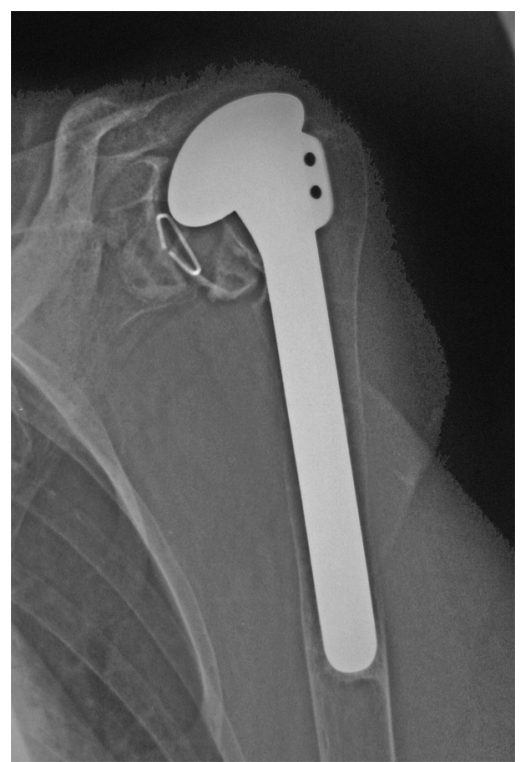

Fig. 1

Radiograph of a total shoulder replacement showing proximal humeral migration, glenoid loosening and a periosteal reaction at the tip of the humeral component.

one of whom had grade-5 loosening of the glenoid and subsidence of the humeral component. This had been noted at 9.5 years, but her pain had recently increased.

Complications. Of the surviving shoulders, two had intraoperative fractures. One patient who had an intraoperative fracture of the glenoid had only an incomplete radiolucent line around the keel of the glenoid at the latest follow-up but required re-operation to remove a Kirschner-wire from the glenoid. One of the humeral components perforated the lateral wall of the humerus intraoperatively. This prosthesis did not have any radiological changes during follow-up although the glenoid component showed progression to grade 5 .

Five patients from the original series underwent revision surgery. One had a post-operative rotator-cuff tear and one an infection. Both died later from unrelated causes. In another revision was performed for painful aseptic loosening of both components. In the remaining two the reasons for revision were unclear.

\section{Discussion}

There have been many short- and mid-term studies ${ }^{1-3,9,10}$ on TSR and hemiarthroplasty in mixed groups of patients, but no long-term report of more than 12.3 years. ${ }^{4}$ Total shoulder replacement fares better than hemiarthroplasty in terms of long-term relief from pain, function and risk of revision ${ }^{5}$ with loosening of the glenoid component as the most common cause of failure. ${ }^{6}$

As many as $94 \%$ of keeled glenoid replacements are reported to have early radiolucent lines. ${ }^{14}$ This rate increases with follow-up, ${ }^{15}$ but is not always clinically relevant as it does not always indicate loosening ${ }^{6,7}$ and is associated with a low rate of revision and few symptoms. Our patients showed good early results, ${ }^{1,2}$ but concern was expressed over the long-term progression of the radiolucent lines which were present in 23 of the $37(62 \%)$ shoulders at 9.5 years and 11 of 14 shoulders $(78.6 \%)$ at the latest follow-up. Two glenoid components had earlier been noted to have complete radiolucent lines, of which one progressed to grade-5 loosening. Poor glenoid bone stock and proximal migration of the humeral head were associated with early radiolucency around the glenoid component. ${ }^{2-4,16}$ In the current follow-up, glenoid loosening seemed to be associated with proximal humeral migration and was unrelated to the glenoid bone stock at the time of operation. This sug- 
gests that glenoid loosening is a multifactorial process, with early loosening linked to glenoid bone stock and late loosening to proximal humeral migration and the inevitable failure of the rotator cuff in patients with rheumatoid arthritis, ${ }^{2,3,7,16}$ leading in turn to loosening of the humeral component.

Changes in joint kinematics have a long-term influence on glenoid loading. ${ }^{17}$ Loosening is thought to occur because of eccentric loading and a subsequent 'rockinghorse' effect on the glenoid component by a superiorly subluxated humeral head. ${ }^{3,8,18}$ In our earlier report, ${ }^{2} 57 \%$ of humeral heads had migrated proximally and all have now done so. This migration is exacerbated by instability, cuff deficiency and loading on the joint, ${ }^{8}$ which are common in patients with rheumatoid arthritis.

Proximal migration also leads to eccentric loading of the humeral head which can cause the component to toggle. This resulted in an endosteal reaction at the tip of ten of the components. Other studies have shown loosening of more than $40 \%$ of press-fit humeral components at follow-up at $7.7,,^{3,10} 9.5^{2}$ and $12.3^{4}$ years. The routine use of cement for humeral components was therefore recommended. However, since there is evidence that complete radiolucent lines around humeral components are not associated with migration, ${ }^{19}$ the significance remains unclear, even although Cil et al ${ }^{15}$ found that $100 \%$ of humeral components were loose at revision surgery. We feel that this endosteal reaction is secondary to failure of the rotator cuff and change of kinematics, but has not led to changes in the position of the stem.

We have no retrieval data for the revised glenoid components to demonstrate polyethylene wear. Unlike the hip, marked areas of progressive osteolysis at the humeral neck have not been noted in our or other series although areas of lucency immediately beneath the humeral head are common. ${ }^{20}$ This would be expected if loosening was associated with polyethylene particles. It has been suggested that such particles from glenoid wear are larger, aspherical and less reactive, ${ }^{8}$ thereby indicating a different pattern of aseptic loosening than that seen in joints of the lower limb. In the shoulder, failure of the rotator cuff appears to be a dominant factor in the long-term loosening of the components.

The activities and pain scores of our patients were reasonably well maintained, despite radiolucencies around either component and this corresponds to findings in osteoarthritis. ${ }^{20}$ Sneppen et $a 1^{10}$ noted that at follow-up at 7.7 years patients were asymptomatic despite radiological changes and recommended radiological surveillance. There is a high intraoperative complication rate of almost $30 \%$ associated with revision surgery, ${ }^{15}$ with particular difficulties related to poor bone stock and soft tissues ${ }^{14}$ which are prevalent in patients with rheumatoid arthritis and must be considered when planning revision surgery. In our series, patients were still functioning well at 19.8 years, albeit with gradual decline from 9.5 years.
In summary, we noticed progression of radiolucencies around the glenoid component, along with proximal humeral migration and loosening of the humeral component in patients who had a TSR for rheumatoid arthritis. We suggest that failure of the rotator cuff is the dominant feature. In these elderly, low-demand patients, the range of movement was preserved. Most had little or no pain from the shoulder, could sleep undisturbed and attend to personal care, despite radiological evidence of probable loosening of the components and failure of the rotator cuff. This suggests that this procedure is a successful and durable operation for patients with rheumatoid arthritis.

No benefits in any form have been received or will be received from a commercial party related directly or indirectly to the subject of this article.

\section{References}

1. Kelly IG, Foster RS, Fisher WD. Neer total shoulder replacement in rheumatoid arthritis. J Bone Joint Surg [Br] 1987;69-B:723-6.

2. Stewart MPM, Kelly IG. Total shoulder replacement in rheumatoid disease: 7 - to 13-year follow-up of 37 joints. J Bone Joint Surg [Br] 1997;79-B:68-72.

3. Sojbjerg J0, Frich LH, Johannsen HV, Sneppen 0. Late results of total shoulder replacement in patients with rheumatoid arthritis. Clin Orthop 1999;366:39-45.

4. Sperling JW, Cofield RH, Rowland CM. Neer hemiarthroplasty and Neer total shoulder arthroplasty in patients fifty years old or less. J Bone Joint Surg [Am] 1998;80-A:464-73.

5. Sperling JW, Cofield RH, Schleck C, Harmesen WS. Total shoulder arthroplasty versus hemiarthroplasty for rheumatoid arthritis of the shoulder: results of 303 consecutive cases. J Shoulder Elbow Surg 2007;16:683-90.

6. Klepps S, Chiang AS, Miller S, et al. Incidence of early radiolucent glenoid lines in patients having total shoulder replacements. Clin Orthop 2005;435:118-25.

7. Writh MA, Rockwood CA. Complications of total shoulder-replacement arthroplasty. J Bone Joint Surg [Am] 1996;78-A:603-16.

8. Matsen FA 3rd, Clinton J, Lynch J, Bertelsen A, Richardson ML. Glenoid component failure in total shoulder arthroplasty. J Bone Joint Surg [Am]2008;90-A:885-90.

9. Torchia ME. Total shoulder arthroplasty with the Neer II prosthesis: long term results. J Shoulder Elbow Surg 1997;6:495-505

10. Sneppen 0, Fruensgard S, Johannsen HV. Total shoulder arthroplasty in rheumatoid arthritis: proximal migration and loosening. J Shoulder Elbow Surg 1996;5:47-52.

11. Larsen A, Dale K, Eek M. Radiographic evaluation of rheumatoid arthritis and related conditions by standard reference films. Acta Radiol Diagn (Stockh) 1997;18:481-91.

12. Friedman RJ, Thornhill TS, Thomas WH, Sledge CB. Non-constrained total shoulder replacement in patients who have rheumatoid arthritis and class-IV function. J Bone Joint Surg [Am] 1989;71-A:494-8.

13. Lazarus MD, Jensen KL, Southworth C, Matsen FA 3rd. The radiographic evaluation of keeled and pegged glenoid component insertion. J Bone Joint Surg [Am] 2002;84-A:1174-82.

14. Raiss $\mathbf{P}$, Aldinger PR, Kasten $\mathbf{P}$, Rickert $\mathbf{M}$, Loew $\mathbf{M}$. Total shoulder replacement in young and middle-aged patients with glenohumeral osteoarthritis. J Bone Joint Surg [Br] 2008;90-B:764-9.

15. Cil A, Veillette CJH, Sanchez-Sotelo J, et al. Revision of the humeral component for aseptic loosening in arthroplasty of the shoulder. J Bone Joint Surg [Br] 2009;91B:75-81

16. Lehtinen JT, Belt EA, Kauppi MJ, et al. Bone destruction and upward migration, and medialisation of rheumatoid shoulder: a 15 year follow-up study. Ann Rheum Dis 2001;60:322-6.

17. von Eisenharte-Rothe R, Muller-Gerbel M, Weidemann E, Englmeier KH, Graichen $\mathbf{H}$. Functional malcentering of the humeral head and asymmetric long-term stress on the glenoid: potential reasons for glenoid loosening in total shoulder replacement. J Shoulder Elbow Surg 2008;17:695-702

18. Boileau P, Sinnerton RJ, Chuinard C, Walch G. Arthroplasty of the shoulder. Bone Joint Surg [Br] 2006;88-B:562-75.

19. Sanchez-Sotelo J, Wright TW, O'Driscoll SW, Cofield RH, Rowland CM. Radiographic assessment of uncemented humeral components in total shoulder arthroplasty. J Arthroplasty 2001;16:180-7.

20. Zilber S, Radier C, Postel JM, et al. Total shoulder arthroplasty using the superior approach: influence on glenoid loosening and superior migration in the long-term follow-up after Neer II prosthesis installation. J Shoulder Elbow Surg 2008;17:554-63. 